\title{
STUDIES ON AN UNCOMPLICATED CORYZA OF THE DOMESTIC FOWL
}

\author{
IX. The Cooperative Action of Hemophilus gallinarum and \\ THE Coccobacilifform Bodies IN THE CORYza OF \\ Rapid Onset and Long Duration
}

By JOHN B. NELSON, PH.D.

(From the Department of Animal and Plant Pathology of The Rockefeller Institute for Medical Research, Princeton, New Jersey)

(Received for publication, March 5, 1938)

In an earlier paper on fowl coryza it was noted that the disease produced by the intranasal injection of normal birds with exudate from the upper air passages of naturally infected fowl could be divided into three types. Type I was characterized by a rapid onset and short duration, type II by a slow onset and long duration, and type III by a rapid onset and long duration (1).

A Gram-negative bacillus corresponding to the hemophilic bacterium originally described by De Blieck was regularly isolated from birds infected with type I coryza $(2,3)$. There is reason to believe that this bacillus, now known as Hemophilus gallinarum, was the only etiologic factor involved. The relation of $H$. gallinarum to the type III coryza with which it was also associated was less clearly defined. Recently isolated cultures were invariably infective but produced a coryza of short duration in contrast to the prolonged course of the coryza produced by exudate. Confusing irregularities were also observed following the injection of exudate in birds which had recovered from the coryza produced by $\boldsymbol{H}$. gallinarum.

We had previously suggested that the differences between the coryzas produced by $\boldsymbol{H}$. gallinarum and type III exudate might be referable to a degradation in virulence induced by artificial cultivation of the bacillus (1). This suggestion was made before the etiology of type II coryza had been worked out. With the demonstration that this type was caused by minute Gram-negative cells termed coccobacilliform bodies, another explanation was offered (4): namely, that the type III coryza might be due to a mixed infection with these 
bodies and $H$. gallinarum. Observations bearing on this association in relation to the etiology of the coryza of rapid onset and long duration are here presented.

\section{The Demonstration of Coccobacilliform Bodies in Exudate from Type III Coryza.}

The first evidence that coccobacilliform bodies were associated with type III coryza was obtained from birds injected with exudate after recovery from the coryza produced by $H$. gallinarum in pure culture.

Strains of types II and III have been maintained since the fall of 1933 in birds of known history by the serial passage of exudate originally secured from natural cases. In these two groups of birds which are held under strict quarantine in separate units the two types have retained their respective characteristics throughout the long series of transfers. The exudate and the cultures of the two infective agents used in the following experiments were obtained from these birds.

Five normal birds were injected intranasally by way of the palatine cleft with approximately $0.5 \mathrm{cc}$. of the fluid from the base of a 48 hour horse blood agar culture of $\boldsymbol{H}$. gallinarum (the 4th subculture since isolation). The birds in this experiment and the succeeding ones were Rhode Island Reds, 8 to 12 weeks old, from a flock reared at the Institute. They were placed in single cages in the same isolation unit with the birds infected with the stock strain of type III coryza. This quarantine unit is large and has a separate compartment at one end which affords partial isolation. The injected birds were held in this compartment and examined daily. 2 to 3 weeks after the nasal discharge had subsided they were injected with $0.5 \mathrm{cc}$. of a broth suspension of type III exudate. The reaction produced by this exudate is so constant that only a single control was included. Several days after the appearance of a discharge the birds were killed and autopsied. The nasal exudate was examined culturally and microscopically and tested for infectivity by injection in normal birds.

As indicated in Table $I$ the 5 birds injected with $H$. gallinarum showed a nasal discharge after a brief incubation period of 1 to 3 days. The duration of the nasal discharge was also short, 5 to 13 days. The injection of type III exudate after recovery from the initial coryza was followed by a second coryza which was of slow onset, 13 to 28 days. The bacteriological examination of exudate from these birds, made 1 to 5 days after the onset of the second coryza, failed to demonstrate $H$. gallinarum, but did indicate the presence of coccobacilliform bodies. The infectivity tests showed a coryza of slow onset, 16 to 23 days, and of long duration, 34 to 58 days. The single 
normal bird injected with type III exudate developed a coryza on the 2nd day, $H$. gallinarum being recovered from the nasal exudate.

The inoculation of tissue cultures (test tubes containing minced 10 day chick embryo tissue suspended in $5.0 \mathrm{cc}$. of Tyrode's solution) with exudate from the sinuses of the 5 birds employed in the infectivity tests resulted in the isolation of one pure strain of the coccobacilliform bodies. This strain was carried through a number of subcultures and compared with a stock culture originally obtained from the type II coryza. The morphology, cultural behavior, and infectivity of the two strains were practically identical.

TABLE I

The Reaction to Type III Exudate after Recovery from Infection with Hemophilus gallinarum

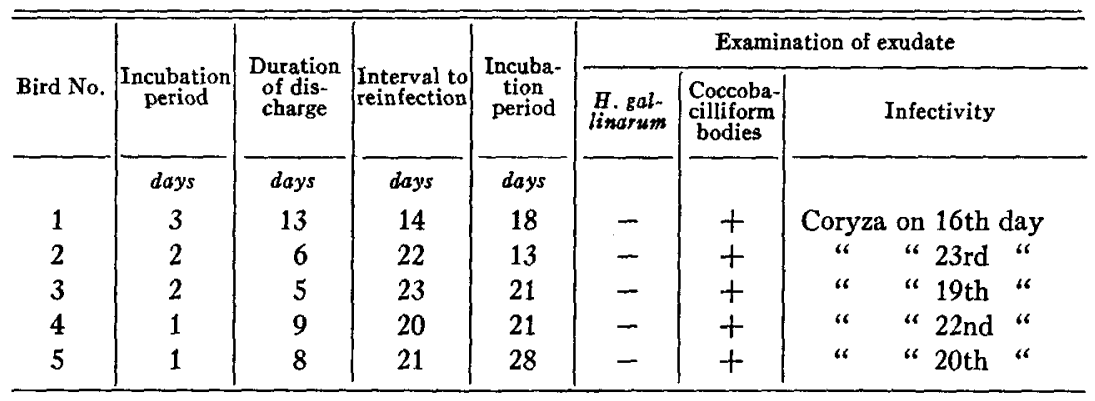

Coccobacilliform bodies were generally demonstrable, thereafter, in birds infected with type III exudate. In a few birds, particularly those with a coryza of long standing, a diagnosis was impossible because of the huge numbers of miscellaneous bacteria. The morphological characteristics of these bodies are so definite that cultivation is not necessary for their identification. The microscopic examination, in addition to demonstrating that coccobacilliform bodies were present in type III exudate, brought out the unexpected finding that they were present as early as the 1st day after injection.

It was now established that exudate from birds infected with the coryza of rapid onset and long duration contained both $\mathrm{H}$. gallinarum and the coccobacilliform bodies. It was demonstrated, moreover, that both agents were present throughout the entire course of the 
disease. This observation was contrary to the accustomed behavior of the two agents when injected in pure culture. Several years' experience with different strains of the specific bodies had indicated that their development in the nasal passages of the host was slow. They were rarely demonstrable before the 10 th day and frequently did not provoke a reaction from the host before the 2nd or 3rd week. In birds infected with type III exudate they were demonstrable as early as the 1st day after injection. Concerning $H$. gallinarum it was known to be recoverable throughout the course of the coryza, but pure cultures were rarely able to maintain their existence in the host for a period longer than 2 weeks and frequently succumbed after a residence of several days. The obvious approach to a solution of this problem was to determine the behavior of an artificial mixture of the two infective agents in the nasal passages of susceptible birds.

The Combined Action of Hemophilus gallinarum and the Coccobacilliform Bodies in Susceptible Fowl

Five successive experiments were carried out on the combined action of the two infective agents. These experiments were unavoidably prolonged, requiring a little over a year for completion. This served, however, to bring them into a rough relationship with the different seasons.

A different cuiture of $B$. gallinarum was used in each experiment, all of them being isolated from the stock strain of type III coryza. Four cultures of the specific bodies were employed; one isolated from the type III coryza, the others from type II. In preparing suspensions for injection the supernatant was removed from a 24 to 48 hour tissue culture of the coccobacilliform bodies, the sediment finely ground, and suspended in the supernatant. An equal volume of fluid from the base of 24 to 48 hour horse blood agar slants of $B$. gallinarum was added and roughly $0.5 \mathrm{cc}$. of the mixture injected through the cleft. The infectivity of each component was checked by adding an equal volume of Tyrode's solution and injecting separately. H. gallinarum was reisolated at intervals from the birds injected with the mixed suspension and tested. It was often difficult to isolate $H$. gallinarum by plating exudate from birds with a prolonged coryza because of overgrowths by Bacillus proteus and other bacteria. If this occurred exudate was injected into a normal bird from which the organism was readily recovered. Films of exudate were also made at intervals and Gram stained for detection of the specific bodies. 
All of the 20 birds employed in the five experiments on the combined action of the two infective agents showed a coryza of rapid onset; the incubation periods being 1 day in 11 cases, 2 days in 8 , and 3 days in a single instance. The birds were kept under observation for 30 to 80 days and without exception showed a continuous nasal discharge. 3 birds died, after 30,40 , and 70 days. 2 others were so severely affected that they were killed, after 41 and 45 days. In 14 individuals the duration of the coryza was at least 2 months.

TABLE II

Duration of Coryza Produced by Hemophilus gallinarum Alone and in Combination with the Coccobacilliform Bodies

\begin{tabular}{|c|c|c|c|c|}
\hline \multirow{2}{*}{ Infective agent } & \multicolumn{2}{|c|}{ Experiment 1} & \multicolumn{2}{|c|}{ Experiment 2} \\
\hline & Bird No. & $\begin{array}{c}\text { Duration of } \\
\text { coryza }\end{array}$ & Bird No. & $\begin{array}{c}\text { Duration of } \\
\text { coryza }\end{array}$ \\
\hline \multirow{4}{*}{$\begin{array}{l}\text { H. gallinarum cultures used in } \\
\text { the mixture }\end{array}$} & & days & & days \\
\hline & 1 & 3 & 12 & 5 \\
\hline & 2 & 5 & 13 & 7 \\
\hline & 3 & 15 & 14 & 4 \\
\hline \multirow{5}{*}{$\begin{array}{l}\text { H. gallinarum and coccobacil- } \\
\text { liform bodies }\end{array}$} & 4 & $>45$ & 15 & 70 \\
\hline & 5 & $>56$ & 16 & $>70$ \\
\hline & 6 & $>56$ & 17 & $>70$ \\
\hline & 7 & $>41$ & 18 & $>70$ \\
\hline & & & 19 & $>70$ \\
\hline \multirow{5}{*}{$\begin{array}{l}\text { H. gallinarum cultures isolated } \\
\text { from birds infected with the } \\
\text { mixture }\end{array}$} & 8 & 4 & 20 & 5 \\
\hline & 9 & 5 & 21 & 4 \\
\hline & 10 & 8 & 22 & 5 \\
\hline & 11 & 16 & 23 & 10 \\
\hline & & & 24 & 3 \\
\hline
\end{tabular}

At autopsy all of these birds were emaciated and showed evidence of constitutional disorder. One bird recovered after a nasal discharge which lasted 44 days. With the exception of this case, coccobacilliform bodies were demonstrable at autopsy. $H$. gallinarum was recovered by subinjection from 10 of the birds which showed a nasal discharge for 56 days or longer. The birds which were injected for these isolations also showed coccobacilliform bodies as early as the 1 st or 2nd day. 
With several exceptions which will be discussed later, the birds injected with $H$. gallinarum alone showed the usual coryza of rapid onset and short duration. In 8 birds the duration was 7 days or less, in 5 it was 10 to 16 days. Most of these birds were killed and autopsied 1 to 2 weeks after recovery. Save for the above noted exceptions the nasal passages and sinuses were normal. Similar findings were obtained with cultures of $H$. gallinarum isolated at intervals from the test birds. The summary of two experiments in Table II affords a comparison of the infectivity of $H$. gallinarum alone and in combination with the coccobacilliform bodies.

Infectivity tests were also made with the several cultures of the coccobacilliform bodies. These tests which were carried out in a separate quarantine unit did not always coincide with the combined experiments in point of time. Some of the birds showed an inapparent coryza which was detected only at autopsy. If a nasal discharge was produced it was always after an incubation period of 2 weeks or longer.

\section{Transmission of the Coccobacilliform Bodies by Indirect Contact}

One bird of a group of 4 injected in June, 1937, with a pure culture of $H$. gallinarum continued to show a nasal discharge long after the other 3 had recovered. It was known that $H$. gallinarum occasionally produced a coryza of 3 to 4 weeks' duration, and the case was not regarded as unusual. The bird was reexamined in September, 78 days after injection, and still showed a nasal discharge, though slight and unilateral. On Sept. 13, the discharge having subsided, the bird was killed and autopsied. The nasal passages were normal, but in one sinus there was a large rubbery plug of exudate which showed Gram granules suggestive of the coccobacilliform bodies. A normal bird injected with this material developed a coryza with very distinct specific bodies on the 3rd day. At monthly intervals two additional passages were carried out. All of the birds showed a coryza of at least 8 weeks' duration, the exudate containing both infective agents. Through January, 1938, four additional cases of prolonged coryza were observed in birds originally injected with $H$. gallinarum alone, the specific bodies being again demonstrated in the nasal exudate. Although carriage of the coccobacilliform bodies by indirect contact 
had not been observed in the past, it is believed that transfer in this way was responsible for their presence in the birds infected with $H$. gallinarum, development being favored, as it was not in the case of type II coryza, by that organism.

\section{DISCUSSION}

The results of the experiment on the injection of type III exudate, following recovery from. the coryza produced by $H$. gallinarum in pure culture, are illustrative of a number of such tests made from time to time with different strains. Recovered birds usually showed a temporary resistance to reinfection with cultures of the organism, regardless of the strain. If protection was not afforded, the reinjection of $H$. gallinarum was followed by a coryza of rapid onset and by recovery of the organism from the nasal passages. The injection of exudate was usually followed by a second coryza which in the case of the present strain was characterized by a long incubation period and the absence of $H$. gallinarum. It was impossible to explain this behavior on the basis of a lowered virulence resulting from the artificial cultivation of $H$. gallinarum. The disease produced in the recovered birds closely resembled the type II coryza, and coccobacilliform bodies similar to the etiologic agent of the latter type were ultimately demonstrated in the nasal discharges.

Although the present strain of type II coryza had been maintained by artificial passage for 4 years there was no reason to doubt that both $H$. gallinarum and the coccobacilliform bodies had been present from the beginning. The apparent fact that two infective agents were present in infected birds did not immediately clarify the situation. Both agents were demonstrable throughout the entire course of the coryza, which frequently began on the 2nd day after injection and lasted for at least 2 months. This observation disagreed with the behavior of the two agents in pure culture. The coccobacilliform bodies were rarely demonstrable before the 2 nd week following injection and $B$. gallinarum was rarely recoverable after the 2 nd week.

Subsequent investigation showed that these two sets of observations were not incompatible. Pure cultures of the infective agents were mixed, and the effect of the mixture compared with that of each component. The pure cultures behaved in the accustomed way; 
the mixture resulted in a coryza similar to that produced by type III exudate. The onset was rapid, the course prolonged, and both agents were generally demonstrable throughout its entire course.

The apparent explanation of this situation is that $\boldsymbol{H}$. gallinarum and the coccobacilliform bodies when present together in the nasal passages of the host cooperate in producing an effect that neither is able to accomplish alone. It is clearly a synergistic, or in the older sense (since both agents benefit by the association) a symbiotic reaction. The rapidly multiplying $H$. gallinarum creates a favorable environment for the immediate development of the coccobacilliform bodies. The latter evidently by reason of their tendency to persist in the host prolong the residence of $H$. gallinarum. It is probable that the beneficial influence of $B$. gallinarum is the factor which makes possible the occasional development of the coccobacilliform bodies transmitted by indirect contact, in normal birds the number which gain entrance to the nasal passages being below the threshold for survival.

The cooperative association of $H$. gallinarum and the coccobacilliform bodies adequately accounts for the etiology of the present strain of type III coryza. Schalm and Beach have reported observations which indicate that long continued bird to bird passage of $\boldsymbol{H}$. gallinarum, starting with a pure culture, may prolong its residence in the nasal passages (5). It is possible that a similar adaptation may occasionally occur in nature. Hence, a generalization applicable to this type of coryza is not warranted at present.

\section{SUMMARY}

Coccobacilliform bodies were regularly demonstrable, in addition to $H$. gallinarum, in exudate from birds infected with a passage strain of the coryza of rapid onset and long duration (type III). Both agents were present throughout the entire course of the disease.

The characteristics of type III coryza were reproduced by injecting a mixture of the two agents. The behavior of each component was altered by the association, indicative of a synergistic relation.

Evidence that the coccobacilliform bodies might occasionally de- 
velop in birds infected with $H$. gallinarum following transfer by indirect contact was also obtained.

The combined action of the two infective agents adequately accounts for the etiology of this particular strain of type III coryza.

\section{BIBLIOGRAPHY}

1. Nelson, J. B., J. Exp. Med., 1933, 68, 297.

2. De Blieck, L., Vet. J., 1932, 88, 9.

3. Nelson, J. B., J. Exp. Med., 1933, 68, 289.

4. Nelson, J. B., J. Exp. Med., 1936, 63, 515.

5. Schalm, O. W., and Beach, J. R., Poultry Sc., 1936, 15, 473. 